\title{
Welcome to the JSAR radiologic-pathologic correlation section
}

\author{
Hiromu Mori \\ Oita University, Oita, Japan
}

Welcome to the JSAR (Japanese Society of Abdominal Radiology) Radiologic-Pathologic Correlation Section. This new section includes 6 case reports [1-6] with beautiful pathological correlation. As the President of the JSAR, it is my great pleasure to introduce where and how these case reports come from.

The JSAR was established in 1990 and now has a 26-year history. From its start, founding members had decided to form a society having both functions of gastrointestinal radiology (so-called GI) and of genitourinary radiology (so-called GU). In addition to this, it was an outstanding decision made by founding members that case reports which were strictly correlated with pathological findings would be the main constituent of its annual meeting, and all oral presentations are subject of comments of invited pathologists. The policy of annual meeting of the JSAR has been requesting presenters of case reports to establish complete pathological correlation in his/her institution. The JSAR invites 5-6 pathologists every year 2 weeks earlier to the annual meeting to check abstracts and pathological images. At the time of presentation, discussion between pathologists and radiologists will make a great and more perfect correlation. Discussion between presenters, pathologists, and experts of both gastrointestinal radiology and genitourinary radiology reaches to the point of diagnostic imaging, and young radiologists can learn a lot from any single case.

Dr. Morton A. Meyers, the previous Editor-in-Chief of Abdominal Imaging, and Dr. C. Daniel Johnson, the current Editor-in-Chief of Abdominal Imaging/Radiology, understood the merit of publication of this JSAR's Radiologic-Pathologic Correlation, although most medical journals had given up to publish case reports because of their relatively low citation. Five papers of the JSAR Radiologic-Pathologic Correlation Section first appeared in a group in Abdominal Imaging in 2015

Correspondence to: Hiromu Mori; email: morihrmu@oita-u.ac.jp
[7-11] and three appeared independently [12-14]. This is the second appearance of 6 papers in a group. Of all 14 papers, eight achieved Uchida Award which commemorated the name of late Dr. Hideo Uchida, the first JSAR President, as the most excellent paper of the year at the JSAR annual meeting, and six other papers were highly regarded in the annual meeting of the JSAR 2014 and 2015.

I hope the readers of Abdominal Radiology will enjoy this JSAR's Radiologic-Pathologic Correlation Section.

\section{References}

1. Hashimoto A, Takahama J, Harada N, et al. (2016) A case of uterine torsion concurrent with a ruptured ovarian endometrial cyst. Abdom Radiol (NY). doi:10.1007/s00261-016-0758-z

2. Hayashi TY, Matsuda I, Hagiwara K, Takayanagi T, Hagiwara A (2016) Massive splenic infarction and splenic venous thrombosis observed in a patient with acute splenic syndrome of sickle cell traits on contrast-enhanced thin-slice computed tomography. Abdom Radiol (NY). doi:10.1007/s00261-016-0786-8

3. Himoto Y, Kido A, Fujimoto K, et al. (2016) A case of pseudomyxoma peritonei: visualization of septa using diffusion-weighted images with low b values. Abdom Radiol (NY) (Review). doi: 10.1007/s00261-016-0697-8

4. Minamiguchi K, Takahama J, Marugami N, et al. (2016) MR findings of Lynch syndrome-related uterine endometrial carcinoma: a case report. Abdom Radiol (NY). doi:10.1007/s00261-016-0721-z

5. Takeuchi M, Matsuzaki K, Bando Y, et al. (2016) A case of polypoid endometriosis with malignant transformation. Abdom Radiol (NY). doi:10.1007/s00261-016-0696-9

6. Sagara Y, Matsumoto S, Oga M, et al. (2016) MDCT features of strangulated ileus due to an appendix epiploica of the sigmoid colon: four cases and a review of the literature. Abdom Radiol (NY). doi:10.1007/s00261-016-0835-3

7. Tsuboyama T, Yamamoto K, Nakai G, et al. (2015) A case of gastric-type adenocarcinoma of the uterine cervix associated with lobular endocervical glandular hyperplasia: radiologic-pathologic correlation. Abdom Imaging 40(3):459-465

8. Kawai Y, Nakamichi R, Kamata N, et al. (2015) A case of undifferentiated carcinoma of the pancreas mimicking main-duct intraductal papillary mucinous neoplasm (IPMN). Abdom Imaging 40(3):466-470

9. Kaneda S, Fujii S, Nosaka K, et al. (2015) MR imaging findings of mass-forming endosalpingiosis in both ovaries: a case report. Abdom Imaging 40(3):471-474

10. Yamada K, Takahata A, Ichijo Y, et al. (2015) A case of testicular seminoma in persistent Mullerian duct syndrome with transverse testicular ectopia. Abdom Imaging 40(3):475-479 
11. Takeuchi M, Matsuzaki K, Harada M (2015) MR manifestations of uterine polypoid adenomyoma. Abdom Imaging 40(3):480-487

12. Ishii $\mathbf{M}$, Kazaoka J, Fukushima J, et al. (2015) A case of $\beta$-cateninpositive hepatocellular adenoma with MR imaging sign of diffuse intratumoral fat deposition. Abdom Imaging 40(6):1487-1491

13. Yoneda N, Matsui O, Ikeno H, et al. (2015) Correlation between Gd-EOB-DTPA-enhanced MR imaging findings and OATP1B3 expression in chemotherapy-associated sinusoidal obstruction syndrome. Abdom Imaging 40(8):3099-3103

14. Toshima F, Inoue D, Yoshida K, et al. (2016) Adenosquamous carcinoma of pancreas: CT and MR imaging features in eight patients, with pathologic correlations and comparison with adenocarcinoma of pancreas. Abdom Radiol (NY) 41(3):508-520 